\title{
Utilizing Instagram for Brand Management in Sport
}

\author{
Shahrzad Nayyeri, Ph.D. \\ Assistant Professor \\ Management and Technology Expansion Studies Center, Tarbiat Modares University \\ Email: Sh.nayyeri@modares.ac.ir \\ Address: Jalal AleAhmad, Nasr, Tehran, Iran, 14115-111
}

\author{
Vajiheh Javani \\ Assistant Professor \\ Department of Sport Management, University of Tabriz \\ Email: V.javani@tabrizu.ac.ir \\ Address: 29 Bahman Blvd., Tabriz, Iran, 5166616471
}

\begin{abstract}
Social networks provide a direct path for organizations to communicate with consumers and enable them to represent a clear picture of their brands. Todays, Professional sport teams utilize social networking platforms for branding goals, so that most professional sport teams are currently active in online social networks. Meanwhile, Instagram's popularity, along with its ability to share media content, has made it an effective tool for sport teams. Research has shown that visual content, such as images and videos shared on Instagram, will increase the participation of sports fans in social networks that is beyond the scope of viewing and reading and in fact relates to the level of cognitive, emotional and behavioral support that they engage with a particular brand. This study aims to investigation of utilizing Instagram for Brand management in professional sport teams. For gathering data qualitative content analysis was conducted on posts of Instagram page of Tehran Esteghlal F.C. the findings shows that brand elements of its Instagram page including product-related factors (team success, star player and head coach), and non-product related factors (brand mark, management, club's history and tradition, club's culture and values, event's image, sponsor and fans). Therefore, Instagram is useful tool for building brand image and interaction with fans.
\end{abstract}

Keywords: Brand attribute; Instagram; Sport teams; Social network. 